\section{Actualización de Criterios Diagnósticos y Tratamiento de la Neuralgia del Trigémino}

\author{
Update of Diagnostic and Treatment of Trigeminal Neuralgia
}

\begin{abstract}
Resumen
La neuralgia del trigémino (NT) es un cuadro doloroso neuropático que compromete una o más ramas del nervio trigémino. El dolor producido por la NT es descrito como un dolor agudo muy intenso, lancinante o punzante, a manera de descarga eléctrica que se presenta usualmente de forma unilateral y que recorre todo el trayecto del nervio comprometido. Es importante realizar el diagnóstico oportuno de los cuadros de NT para poder diferenciarla adecuadamente de algunos cuadros odontogénicos dolorosos y evitar que el paciente reciba tratamientos innecesarios. La primera línea de tratamiento de la NT es la terapia farmacológica, en la cual se utiliza como fármaco de primera elección a la carbamazepina. La segunda línea de tratamiento de la NT es la terapia no farmacológica donde destacan diversas opciones de tratamiento quirúrgico: etiológico o sintomático.
\end{abstract}

Palabras claves: neuralgia del trigémino, dolor neuropático, diagnóstico, tratamiento, carbamazepina.

\section{Abstract}

Trigeminal neuralgia (TN) is a painful neuropathic condition that involves one or more branches of the trigeminal nerve. The pain produced for the TN is described as a very intense acute pain, stabbing or shooting, as an electric shock that usually occurs of form unilateral and that goes all the way of the nerve involved. It is important to make the opportune diagnosis of the condition of TN for adequately differentiate of some odontogenic painful conditions and to prevent that the patient receive unnecessary treatment. The first line of treatment of the TN is the pharmacological therapy, in which is used as a drug of first choice to carbamazepine. The second line of treatment of TN is the non-pharmacological therapy, where include various options of surgical treatment: etiologic or symptomatic.

Keywords: trigeminal neuralgia, neuropathic pain, diagnostic, treatment, carbamazepine.
Artículo DE Revisión

\section{Víctor Chumpitaz Cerrate', Cristian Sayán Sánchez², Eliberto Ruíz Ramírez², César Franco Quino ${ }^{3}$, Juan Eche Herrera ${ }^{3}$, Victoria Caldas Cueva ${ }^{3}$, Yuri Castro Rodríguez ${ }^{3}$, Carlos Erazo Paredes $^{2}$}

\footnotetext{
1 Profesor Auxiliar del Departamento de Ciencias Básicas de la Facultad de Odontología UNMSM.

2 Bachiller en Odontología de la Facultad de Odontología UNMSM.

3 Estudiante de Odontología de la Facultad de Odontología UNMSM.
}

Correspondencia:

Dr. CD. Victor Manuel Chumpitaz Cerrate.

Facultad de Odontología, Universidad Nacional Mayor de San Marcos.

Correo electrónico: vchumpitazc@unmsm.edu.pe

\section{Introducción}

La Neuralgia del Trigémino (NT) es un dolor neuropático cuya etiología es variada, la más aceptada es por compresiones vasculares a nivel del tronco cerebral. El dolor se presenta de forma espontánea, generalmente en zonas gatillo ante estímulos térmicos (zona cutánea, gingival, labial), táctiles o al masticar, hablar, comer, sonreír o bostezar Generando discapacidad para actividades fundamentales y cotidianas como la fonación, masticación, cepillado dental, afeitado o la expresión facial. La NT afecta a 4,3 de cada 100.000 habitantes cada año, con mayor prevalencia en mujeres. Puede iniciarse a cualquier edad, aunque la mayoría de formas idiopáticas se presentan en mayores de 50 años. ${ }^{1}$ La necesidad de recomendar un tratamiento farmacológico, depende de la severidad del cuadro, frecuencia, intensidad y recurrencia de las crisis, que generalmente aumentan con el paso del tiempo. Existen numerosos estudios epidemiológicos, en los que se demuestra que la carbamazepina es el fármaco más utilizado y más eficaz, aunque puede presentar efectos adversos como alteraciones de las funciones cerebrales superiores. En algunos estudios, lamotrigina ha demostrado ser más efectiva, sin embargo, las reacciones cutáneas son más frecuentes. Gabapentina y pregabalina han demostrado buenos resultados y podrían indicarse en combinación con carbamazepina o en casos resistentes a ésta. La cirugía se reserva para casos resistentes al tratamiento médico, o en los que se evidencia una alteración orgánica. ${ }^{2,3}$ Debemos resaltar el rol importante que cumple el odontólogo al realizar un adecuado exa-
Fecha de recepción: 08 de abril

Fecha de aceptación: 14 de junio men clínico y diagnóstico estomatológico para diferenciar cuadros dolorosos de origen dentario que tienen sintomatología similar a la NT.

\section{Metodología}

Se realizó una revisión de la bibliografía publicada en artículos de revistas especializadas y tesis de postgrado, desde el ańo 2000 hasta la actualidad, a partir de los cuales se seleccionó la información mejor documentada, más relevante y relacionada específicamente a los criterios para realizar un adecuado diagnóstico y tratamiento de la neuralgia del trigémino. La información obtenida al finalizar la revisión fue organizada en tres secciones: (a) criterios diagnósticos, (b) tratamiento farmacológico, (c) tratamiento no farmacológico. 


\section{(a) Criterios diagnósticos}

Clínicamente la NT se caracteriza por un dolor agudo, tipo descarga eléctrica, unilateral, que compromete a 1, 2 ó 3 ramas del nervio trigémino. Se presenta en episodios de corta duración, múltiples a lo largo del día, alternando con periodos libres de dolor. Generalmente existe un punto de descarga o punto gatillo a nivel facial, frecuentemente ubicado a nivel del surco nasogeniano. En pocas ocasiones, los pacientes definen el dolor como sordo, penetrante y continuo. El dolor puede desaparecer espontáneamente durante meses o años. Sin embargo, en la mayoría de pacientes las crisis son cada vez más frecuentes, más intensas y las características del dolor van cambiando, indicando probablemente que la enfermedad es progresiva, sugiriendo por lo tanto un mecanismo neuropático en su origen. Los casos bilaterales se presentan en 3-4\% de pa-

Tabla 1. Criterios diagnósticos de la Neuralgia del Trigémino

\begin{tabular}{ll}
\hline 1. Localización & Dermatomas trigeminales, generalmente unilateral \\
2. Radiación & Área trigeminal y otros dermatomas \\
3. Características & Agudo, descargas, eléctrico \\
4. Intensidad & Moderado a niveles de suicidio \\
5. Duración & Episodios de dolor de varios minutos, múltiples a lo \\
6. Frecuencia & largo del día \\
7. Factores desencadenantes & Series de episodios de dolor alternados con periodos \\
8. Alivio del dolor & Presión leve \\
9. Factores asociados & Fármacos antineurálgicos \\
\hline
\end{tabular}

Los estudios radiológicos complementarios, que son ineludibles, como la tomografía axial computarizada (TAC) y la resonancia magnética (RNM) cerebral, van encaminados a diferenciar entre una neuralgia esencial o secundaria. La Angio-RNM es la prueba de elección para detectar las compresiones vasculares en la zona del V par a nivel del tronco cerebral. En el diagnostico diferencial se deben buscar condiciones que afecten los senos paranasales, prótesis, abscesos odontogénicos y otras anormalidades de la lengua, encías, maxilar, mandíbula, articulación temporomandibular, enfermedades oculares como glaucoma, celulitis periorbitaria, tumores faciales, enfermedades de oídos, nariz y cuello. ${ }^{5}$

cientes, pero es raro que ambos lados estén activos ¡el lado derecho es el más común?, explicación desde el punto de vista anatómico, por el menor diámetro de los forámenes rotundum y oval en dicho lado, aún en personas sin NT (2). El examen físico y neurológico generalmente es normal, aunque pueden detectarse alteraciones sensitivas a nivel facial y del reflejo corneal en la neuralgia esencial. En la neuralgia secundaria o sintomática, se han observado déficits neurológicos en $47 \%$ de pacientes. ${ }^{4}$

La International Association for the Study of Pain (IASP) definió la NT como un dolor súbito, generalmente unilateral, grave, punzante y de breve duración, en la distribución de una o más ramas del quinto par craneal. ${ }^{4}$

El diagnóstico se basa en la existencia de cuatro de los nueve criterios clínicos descritos por la International Headache Society (IHS). ${ }^{4}$ 
efecto inhibitorio mayor. Se emplea por vía oral en una dosis de $900-3600 \mathrm{mg} /$ día repartidos en 3 administraciones. Hay que tener precaución en la dosificación en pacientes con insuficiencia renal. No son frecuentes las interacciones con otros fármacos, a excepción de los antiácidos. Posee efectos adversos como ataxia, mareos, diplopía, etc. ${ }^{10}$

La lamotrigina actúa modulando el voltaje y la frecuencia de apertura de los canales de sodio dependientes de voltaje y bloquea la entrada del calcio necesaria para la liberación de glutamato. Se considera que este fármaco no es de elección en caso de un ataque agudo de dolor de origen neuropático, ya que causa irritabilidad cutánea, ataxia, diplopía y mareos por lo que su uso debe ser iniciado de manera lenta y escalonada. La dosis es de 200-400 mg/día, una o dos veces al día, sin exceder la dosis de $500 \mathrm{mg} /$ día. $^{11}$

El ácido valproico posee los mejores resultados cuando es usado en combinación con uno o más fármacos. Sus mecanismos de acción incluyen el aumento de la concentración del GABA, bloqueo de canales de calcio, bloqueo de canales de sodio y potasio. La dosis oral es de 500-1500 mg/día, dividido en dos tomas. Está contraindicado en insuficiencia hepática, trastornos de coagulación, trastornos del metabolismo y del ciclo de la urea. Puede producir aumento de peso, alopecia, retraso en el metabolismo de otros fármacos, etc. $^{12}$

La pregabalina modula la permeabilidad de los canales de calcio, lo cual reduce la liberación y disponibilidad de neurotransmisores excitatorios. Posee una mejor farmacocinética comparada con la gabapentina ya que la saturación de su absorción no es dosis-dependiente, sin embargo aún posee efectos adversos como somnolencia, edema periférico y boca seca, entre otros. Logra efectos terapéuticos a dosis que van desde $150 \mathrm{mg}$ a $600 \mathrm{mg}$ por vía oral, 3 veces por día. ${ }^{13}$

Otra sustancia utilizada para el tratamiento de la NT es la toxina botulínica tipo A, que gracias a su efecto antiespasmódico y antinociceptivo puede ayudar a reducir el dolor asociado a la NT, ya que impide la espasticidad muscular relacionada al dolor neuropático y reduce la síntesis de sustancia P, calcitonina y glutamato, lo cual reduce la hipersensibilidad mostrada por las fibras nerviosas afectadas. En el tratamiento de esta patología se usa 100 unidades diluidas en
$2.5 \mathrm{~mL}$ de solución salina de depósito, administrados cada 6 meses. ${ }^{14}$

El leviteracepam, posee un mecanismo de acción desconocido, sin embargo algunos autores sostienen que produce un bloqueo selectivo de los canales de calcio a nivel de los ganglios basales. Proporciona rápido alivio del dolor lo cual permite que el paciente realice sus actividades con normalidad, su absorción no es dosis- dependiente y su farmacocinética es muy predecible ya que su metabolismo es a nivel renal y posee mayor índice terapéutico lo cual nos permite controlar mejor sus efectos adversos. Estos se manifiestan en la terapia adjunta con otros depresores del SNC y son astenia, somnolencia, depresión, etc. Sin embargo se requieren estudios más amplios para demostrar su efectividad real en el tratamiento de la NT, ya que en la actualidad solo ha demostrado una efectividad parcial. La dosis es de $500 \mathrm{mg}$ a $4000 \mathrm{mg} /$ día, divididos en dos dosis. ${ }^{15,16}$

Las vitaminas del complejo $\mathrm{B}$, tiamina (B1), piridoxina (B6) y cianocobalamina (B12), han demostrado ser clínicamente útiles para el tratamiento de algunas condiciones dolorosas como la NT. Los mecanismos de acción de las vitaminas del complejo B en dolor neuropático incluyen efectos sobre la conducción axonal que contribuyen a una analgesia inmediata, inhibición prolongada de receptores a nivel intra o supraespinal, potenciación de la vía de señalización del GMPc, alteración de los niveles intracelulares de glutamato y calcio, y actividad reconstructiva sobre los nervios degenerados lo que contribuye a recuperar la función neuronal y reducir la duración de la hiperalgesia. Si se toma en cuenta la cantidad de tiamina (B1), para el tratamiento de la NT se utilizan dosis de 150-300 mg/ día vía oral, divididos en 3 dosis; o 100 mg c/24-48 horas por vía intramuscular. ${ }^{11,17}$

\section{(c) Tratamiento no farmacológico}

Para el tratamiento de la NT debe establecerse que se trata de una patología incurable, aunque los síntomas pueden ser controlados durante años empleando tratamientos farmacológicos y quirúrgicos. ${ }^{18} \mathrm{El}$ tratamiento quirúrgico constituye la modalidad más efectiva de tratamiento. Debido a que de 25 a 50\% de pacientes no responde al tratamiento farmacológico, se han desarrollado alternativas quirúrgicas como la microdescompresión del nervio trigémino. ${ }^{19}$
El tratamiento quirúrgico de la NT puede ser de dos tipos: el tratamiento etiológico, cuando la causa de la NT tiene una solución quirúrgica (cuando la NT es producida por un tumor o una compresión vascular); y el tratamiento sintomático. Cada vez son más frecuentes los estudios que demuestran excelentes resultados en términos de alivio del dolor y reducción de la duración del mismo con la descompresión microvascular a nivel de la fosa posterior, pero este procedimiento quirúrgico no se puede realizar en personas de avanzada edad. ${ }^{18}$ Los casos farmacorresistentes son candidatos a descompresión microvascular cuando existe buen estado general y una esperanza de vida mayor a 5 ańos. ${ }^{20}$ El tratamiento quirúrgico sintomático está indicado cuando el tratamiento farmacológico ha fracasado, porque no logró controlar adecuadamente el dolor o porque produce efectos secundarios severos. ${ }^{21}$ Actualmente hay varias posibilidades de tratamiento quirúrgico: 1) Descompresión microvascular quirúrgica; 2) Radioterapia estereotáctica; 3) Microcompresión percutánea; 4) Rizólisis percutánea con glicerol; 5) Radiofrecuencia percutánea; 6) Estimulación del ganglio de Gasser con neuromodulación; 7) Rizotomía percutánea; 8) Craneotomía con descompresión neurovascular. ${ }^{12,1}$

\section{Discusión}

La neuralgia del trigémino (NT) es un síndrome caracterizado por dolor facial de tipo lancinante o punzante, intenso, de inicio brusco, duración corta, unilateral y recurrente, localizado en la distribución de una o más ramas del nervio trigémino. 1 La NT es un cuadro doloroso neuropático que compromete una o más ramas del nervio trigémino. La Asociación Internacional para el Estudio del Dolor (IASP) nos brindó el 2011 la última definición sobre dolor neuropático: "dolor causado por una lesión o enfermedad del sistema somato-sensorial". Los mecanismos responsables del dolor persistente que ocurre luego de la injuria neural comprenden alteraciones estructurales y funcionales en el sistema nervioso y se producen en neuronas aferentes primarias y en el SNC. ${ }^{22}$

La alteración de las neuronas sensoriales aferentes primarias puede deberse a liberación de citoquinas inflamatorias por macrófagos y células de Schwann y pérdida de soporte periférico de factores neurotróficos. Se ha demostrado que se modifica la expresión de canales de so- 
dio en neuronas primarias injuriadas. Los canales de sodio Nav1.8 y Nav1.9 sufren down regulation y Nav1.3 sufre up regulation. ${ }^{23}$

La compresión de la raíz trigeminal por una arteria, o más raramente por una vena, como el origen de la NT clásica es demostrable en $90-95 \%$ de pacientes, según reportes hallados en una serie de pacientes que fueron sometidos a descompresión microvascular como tratamiento de la neuralgia. De acuerdo a las guías de manejo de la NT, existe una asociación altamente significativa entre la presencia de compresión neurovascular evidenciada en las imágenes de resonancia magnética y la presencia de la NT. Los cambios morfológicos en el nervio incluyen desviación, distorsión, formación de ranuras y atrofia. ${ }^{24}$

En la actualidad el tratamiento para la NT es inicialmente farmacológico, en el cual, a pesar de la gran variedad de fármacos de los que se dispone en los últimos ańos, la carbamazepina permanece siendo el medicamento de primera elección debido a que ha demostrado una gran efectividad en diversos estudios realizados, llegando incluso a ser más efectiva que algunos de los nuevos fármacos disponibles. ${ }^{6,7}$

En 30\% de los casos el tratamiento médico farmacológico fracasa, debido a que no puede conseguir un adecuado control del dolor producido por las crisis, o por los efectos adversos producidos por los fármacos. En estos casos la interrupción de la vía nerviosa puede ser una opción y esto se puede realizar por diversas técnicas quirúrgicas. Los compuestos químicos neurotóxicos pueden ser inyectados en la periferia del nervio o directamente en el ganglio de Gasser. Los primeros compuestos que se utilizaron para este fin fueron el cloroformo y el ácido asínico. El alcohol fue introducido a inicios del siglo XX y ha sido utilizado extensamente a pesar de que su uso no está libre de complicaciones. Algunos autores describen el uso de la inyección periférica de estreptomicina y/o lidocaína para el tratamiento de la NT, otros autores recomiendan la infusión continua de bupivacaína. Otras técnicas para el control del dolor por NT por una intervención periférica incluyen neurotomía periférica (la cual puede resultar en anestesia permanente), y crioterapia (con muy pocas complicaciones reportadas). Los neurocirujanos prefieren técnicas más radicales como la descompresión microvascular y la termocoagulación por radiofrecuencia. $^{24,25,26}$

\section{Conclusiones}

La NT es un síndrome doloroso neuropático caracterizado por un dolor agudo, tipo descarga eléctrica, unilateral, cuyo diagnóstico se debe basar en un exhaustivo examen clínico.

La mayoría de pacientes con diagnóstico de NT responden adecuadamente al tratamiento farmacológico, siendo la carbamazepina el fármaco que ha demostrado mayor efectividad.

El tratamiento quirúrgico de la NT, etiológico o sintomático, se debe reservar solamente para los pacientes en los cuales el tratamiento farmacológico no ha dado resultados adecuados.

\section{Referencias bibliográficas}

1. Abarca J. Neuralgia del trigémino. Rev Neurocirugía Contemporánea 2007; 1(4): 1-6.

2. Santos F. Neuralgia del trigémino. Arch Neurocien (Mex) 2005; 10(2): 95-104.

3. Kanai A, Saito M, Hoka S. Subcutaneous sumatriptan for refractory trigeminal neuralgia. Headache 2006; 46(4): 577-582.

4. Zakrzewska JM. Trigeminal Neuralgia. Clin Evid 2001; 6: 102936.

5. Nurmikko TJ, Eldridge PR. Trigeminal neuralgia-pathophysiology, diagnosis and current treatment. Br J Anaesth Nurmikko TJ, Eldridge PR. Trigeminal neuralgiapa-thophysiology, diagnosis and current treatment. Br J Anaesth 2001; 87(1): 17-32.

6. Bagheri SC, Farhidvash F, Perciaccante VJ. Diagnosis and treatment of patients with trigeminal neuralgia J Am Dent Assoc 2004; 135: 1713-1717.

7. Balasubramaniam R, Klasser GD, Delcanho R. Trigeminal autonomic cephalalgias: A review and implications for dentistry. J Am Dent Assoc 2008; 139: 1616-1624.

8. Horowitz M, Horowitz M, Ochs M, Carrau R, Kassam A. Trigeminal neuralgia and glossopharyngeal neuralgia: Two orofacial pain syndromes encountered by dentists. J Am Dent Assoc 2004; 135: 14271433.

9. Wiffen P, Collins S, McQuay H, Carroll D, Jadad A, Moore
A. Fármacos anticonvulsivantes para el dolor agudo y crónico (Revisión Cochrane traducida). En: La Biblioteca Cochrane Plus, 2008 Número 4. Oxford: Update Software Ltd. Disponible en: http://www.update-software. com/BCP/BCPGetDocument. asp? DocumentID=CD001133

10. Sarlani E, Grace E, Balciunas B, Schwartz B. Trigeminal neuralgia in a patient with multiple sclerosis and chronic inflammatory demyelinating polyneuropathy. J Am Dent Assoc 2005; 136; 469-476.

11. Navarro A. Revisión farmacológica; Antineuríticos, Atención Familiar, 2006; 13(2): 37-41.

12. Van Kleef M. Trigeminal Neuralgia. World Institute of Pain 2009; 9: 252-259.

13. Obermann M. Efficacy of pregabalin in the treatment of trigeminal neuralgia. Cephalalgia 2007; 28: 174-181.

14. Ngeow W, Nair R. Injection of botulinum toxin type A (BOTOX) into trigger zone of trigeminal neuralgia as a means to control pain, Oral Surg, Oral Med, Oral Pathol, Oral Radiol Endod 2010; 109: e47-e50.

15. Jorns TP. Pilot study to evaluate the efficacy and tolerability of levetiracetam (Keppra_) in treatment of patients with trigeminal neuralgia, European Journal of Neurology 2009;16:740-744.

16. Fisher et al. Trigeminal Neuralgia: Currents Treatments And Future Developments. Expert Opin Emerging Drugs 2003; 8(1): 123143.

17. Wang ZB, Gan Q, Rupert RL, Zeng YM, Song XJ. Thiamine, pyridoxine and cyanocobalamin and their combination inhibit thermal, but not mechanical hyperalgesia in rats with primary sensory neuron injury. Pain 2005; 114: 266-277.

18. Robaina F. Neuralgia del trigémino, revisión del tratamiento médico y quirúrgico. Rev Esp Dolor 2008; 4: 248-256.

19. Delgado C.M., Tamayo A.C., López J.A., Cardona A.V., Granja E., Guevara U. Tratamiento algológico de neuralgia del trigémino, Experiencia de 15 años. Cirugía y cirujanos 2006; 74: 83-88. 
20. Sola R. y Escosa. M. Descompresión microvascular de la neuralgia del trigémino causada por dolicoectasia vertebrobasilar. Revista de Neurología 2001; 32(8): 742745.

21. Peraire, M. Diagnóstico y tratamiento del paciente con neuralgia del trigémino. Neurología 1997; 12: 12-22.

22. Jensen TS, Baron R, Haanpaa M. A new definition of neuropathic pain. Pain 2011; 152: 2204-5.

23. Golan D. Principles of Pharmacology. The Pathophysiologic Basis of Drug Therapy. Third Edition. Philadelphia. Lippincott Williams y Willkins. 2012. Pag 264-283.

24. Lacerda-Leal PR, Roch JA, Hermier M, Nobre-Souza MA, Cristino-Filho G, Sindou M. Structural abnormalities of the trigeminal root revealed by diffusion tensor imaging in patients with trigeminal neuralgia caused by neurovascular compression: A prospective, double-blind, controlled study. Pain 2011; 152: 2357-64.

25. Sirois D, Epler M, Toufani M, Blitzer A. Botulinum Toxin-A (BoNT-
A) in Trigeminal Neuralgia (TN): Outcome of Placebo-Controlled RCT. Oral Surg Oral Med Oral Pathol 2011; 112 (6): e130-e130.

26. Dergin G, Gocmen C, Sener BC. Treatment of trigeminal neuralgia with bupivacaine HCL using a temporary epidural catheter and pain pump: preliminary study. J Craniomaxillofac Surg 2012; 40 (2):124-8. 